\title{
Effects of Charcoal Addition on the Properties of Carbon Anodes
}

\author{
Asem Hussein ${ }^{1,2}$, Mario Fafard ${ }^{2}$, Donald Ziegler ${ }^{3}$ and Houshang Alamdari ${ }^{1,2, *}$ \\ 1 Department of Mining, Metallurgical and Materials Engineering, Université Laval, \\ Québec, QC G1V 0A6, Canada; asem.hussein.1@ulaval.ca \\ 2 NSERC/Alcoa Industrial Research Chair MACE3 and Aluminum Research Centre REGAL, Université Laval, \\ Québec, QC G1V 0A6, Canada; mario.fafard@gci.ulaval.ca \\ 3 Alcoa Primary Metals, Alcoa Technical Center, 100 Technical Drive, Alcoa Center, PA 15069-0001, USA; \\ donald.ziegler@alcoa.com \\ * Correspondence: houshang.alamdari@gmn.ulaval.ca; Tel.: +1-418-656-7666
}

Academic Editor: Hugo F. Lopez

Received: 26 January 2017; Accepted: 13 March 2017; Published: 16 March 2017

\begin{abstract}
Wood charcoal is an attractive alternative to petroleum coke in production of carbon anodes for the aluminum smelting process. Calcined petroleum coke is the major component in the anode recipe and its consumption results in a direct greenhouse gas (GHG) footprint for the industry. Charcoal, on the other hand, is considered as a green and abundant source of sulfur-free carbon. However, its amorphous carbon structure and high contents of alkali and alkaline earth metals (e.g., $\mathrm{Na}$ and $\mathrm{Ca}$ ) make charcoal highly reactive to air and $\mathrm{CO}_{2}$. Acid washing and heat treatment were employed in order to reduce the reactivity of charcoal. The pre-treated charcoal was used to substitute up to $10 \%$ of coke in the anode recipe in an attempt to investigate the effect of this substitution on final anode properties. The results showed deterioration in the anode properties by increasing the charcoal content. However, by adjusting the anode recipe, this negative effect can be considerably mitigated. Increasing the pitch content was found to be helpful to improve the physical properties of the anodes containing charcoal.
\end{abstract}

Keywords: anodes; charcoal; petroleum coke; density; electrical resistivity; mechanical properties

\section{Introduction}

All modern smelters use the Hall-Héroult process to electrolytically reduce the alumina dissolved in molten cryolite. In this process, carbon anodes are used to conduct the high amperage direct electrical current necessary for smelting from the busbar to the electrolyte. Oxygen from alumina is discharged electrolytically and reacts immediately with the carbon anodes, producing gaseous carbon dioxide $\left(\mathrm{CO}_{2}\right)$, which is considered a greenhouse gas (GHG) [1]. A typical prebaked anode consists of approximately $65 \%$ calcined petroleum coke, $20 \%$ recycled anode butts, and $15 \%$ coal tar pitch [2]. Petroleum coke is produced from the heavy residual fractions of crude oil by delayed coking. Due to changes in the oil refining industry (Edwards, 2015), most coke supplies do not comply with anode specifications, which are essentially based on the upper limit of sulfur and heavy metal content, the density, the molecular structure, as well as the mechanical properties [3].

Bio-carbon materials are obtained from the thermal decomposition of biomass, such as wood, in the absence of oxygen. The products of this process take the forms of gases, liquids (bio-oil), and solid char (charcoal). The proportion of each one of the three phases depends on the final pyrolysis temperature and residence time [4]. Being an abundant source of sulfur-free carbon, wood charcoal seems, at first glance, to be an attractive alternative to petroleum coke. Substitution of petroleum coke by charcoal in the anode recipe would reduce fossil $\mathrm{CO}_{2}$ emissions [5]. Furthermore, the very 
low content of vanadium (V) and sulfur (S) in charcoal could allow for the use of petroleum coke with higher $\mathrm{S}$ and $\mathrm{V}$, thus decreasing the raw material cost. However, charcoal is characterized by its amorphous carbon structure and excessive concentrations of inorganic minerals (Ca and $\mathrm{Na}$ ). These undesirable properties result in a material extremely reactive to air and $\mathrm{CO}_{2}$ in addition to its low real and bulk density [5].

There have been very few published works on the use of bio-carbons for anode production [5-8]. Bio-oil distillate residue has been calcined at $1200{ }^{\circ} \mathrm{C}$ in order to produce a bio-renewable calcined coke, and this material has the potential for use in anode applications due to its anisotropic morphology, acceptable specific electrical resistivity, and low concentrations of inorganic minerals [6]. Coutinho et al. [7] produced anode samples by mixing charcoal with bio-pitch. The green anode samples were then baked at $1000{ }^{\circ} \mathrm{C}$ and finally subjected to further heat treatment at $2700{ }^{\circ} \mathrm{C}$. The authors found significant improvements in both the mechanical and electrical properties of bio-anodes as the treatment temperature was increased up to $2700{ }^{\circ} \mathrm{C}$. However, the poor anode properties in addition to the extensive heat treatment temperature make this process difficult to be applied industrially. Monsen et al. [5] investigated the possibility of using charcoal as a substitute for the fine fraction of petroleum coke in carbon anodes for aluminum production. The results showed significant deterioration in mechanical strength, electrical resistivity, and reactivity of the anodes containing charcoal. This was attributed to the low density of charcoal, together with its high inorganic minerals contents.

Hussein et al. [8] tried to improve the properties of raw charcoal via acid washing and heat treatment techniques. Heat treatment at elevated temperatures $\left(1300{ }^{\circ} \mathrm{C}\right.$ or higher) converted its carbon structure into a more ordered one. The calcined charcoal was found to have higher real density and lower specific surface area. In addition, performing an acid washing resulted in a significant depletion in its inorganic mineral content. A combination between acid leaching and calcination reduced both air and $\mathrm{CO}_{2}$ reactivities of charcoal. Air and $\mathrm{CO}_{2}$ reaction profiles of the pre-treated charcoal became comparable to those of the calcined petroleum coke. Substituting a portion up to $10 \%$ of coke with pre-treated charcoal in the anode recipe did not show any negative effect on air and $\mathrm{CO}_{2}$ reactivities of the baked anodes (Hussein, Larachi, Zeigler, \& Alamdari, 2016).

During anode production, pitch plays two roles. First, it covers the coke particles and fills their open pores, transforming the dry particles into a paste amenable to formation into the required shape. Second, during the subsequent baking process, pitch transforms into pitch coke, binding the calcined petroleum coke particles together [2]. Pitch content has a significant influence on anode properties. A minimum pitch content is required to bond the coke aggregates together. However, overpitching should be avoided in order to minimize the volatile loss during the baking process. Adjusting the pitch content in the anode paste is a key factor in achieving the maximum baked anode apparent density and subsequently improving the electrical and mechanical characteristics [2].

In anode plants, the fineness of coke is determined by the Blaine number (BN), which is usually used as an indication of its specific surface area. High Blaine numbers mean a larger surface area; thus, more pitch is required to cover the particle surface, producing an anode paste with acceptable viscosity during the forming process (paste compaction) [9]. Increasing the fineness from 3000 to 6550 Blaine without increasing the pitch/coke mass ratio resulted in the deterioration of both the green anode's and the baked anode's apparent density, electrical resistivity, and mechanical strength [10]. For an increase in the dust fineness of $1000 \mathrm{BN}$, about $1 \%$ more pitch was required to achieve the maximum apparent density of the produced anodes [11]. For a given BN of fine coke in the anode paste, increasing the pitch/coke ratio from 14/100 to 19/100 improved the green density. However, the baked density of these anodes reached a maximum value at a pitch/coke ratio of 16/100 and then decreased by further increasing the pitch content $[9,11]$.

This work attempts to explore the effects of using the pre-treated charcoal as a raw material on the final anode properties. To fulfill the objective, wood charcoal was washed by hydrochloric acid $(\mathrm{HCl})$ and then calcined at the same temperature that the green petroleum coke was calcined, i.e., $1300{ }^{\circ} \mathrm{C}$. 
Anode samples were made by substituting a fine fraction of coke by pre-treated charcoal. The density and mechanical and electrical properties of these anodes were measured and compared to those of the reference anode entirely made of calcined coke and coal tar pitch. As the composition of the anode recipe is changed by the addition of charcoal, the new anode recipe should be optimized. This can be done by changing the particle size distribution and/or by changing pitch/coke mass ratio. During the mixing process, pitch must coat the dry aggregate surface and be able to fill the pores. In this context, the effect of adjusting the pitch content in the anode recipe containing the pre-treated charcoal on the final anode properties was studied.

\section{Materials and Methods}

Industrial calcined petroleum coke provided by Alcoa Inc. (Deschambault, QC, Canada) was crushed and classified into different size fractions as in Table 1 . Fine coke $(<200 \mathrm{mesh})$ with a Blaine number (BN) of 4000 was used in this work. Commercially available maple wood charcoal (Maple Leaf Charcoal Inc., Sainte-Christine d'Auvergne, QC, Canada) was used as the charcoal source. The charcoal was first ball-milled and sieved. A particle size of -400 mesh $(-0.038 \mathrm{~mm})$ was selected for acid washing pre-treatment. The milled charcoal powder was soaked in $1 \mathrm{~mol} \cdot \mathrm{L}^{-1} \mathrm{HCl}(1 \mathrm{~g}$ charcoal per $10 \mathrm{~mL}$ of $\mathrm{HCl}$ ) in a round bottom flask kept in an electrically heated sand bath (Laboratory Craftsmen Inc., Beloit, WI, USA). The process was performed at $65{ }^{\circ} \mathrm{C}$ for $3 \mathrm{~h}$ under continuous stirring. The mixture was then filtered and washed with hot distilled water. The recovered charcoal was then dried overnight at $100{ }^{\circ} \mathrm{C}$. The acid-washed sample was calcined at $1300{ }^{\circ} \mathrm{C}$ in a tubular furnace (MTI Corporation, Richmond, CA, USA) at a constant heating rate of $10 \mathrm{~K} \cdot \mathrm{min}^{-1}$, to the target heat treatment temperature, and then kept at this temperature for $4 \mathrm{~h}$. In order to ensure an inert environment during calcination, the process was performed under a continuous argon flow of $1 \mathrm{~L} \cdot \min ^{-1}$.

Table 1. Size distribution of coke particles.

\begin{tabular}{ccccccccc}
\hline $\begin{array}{c}\text { Particle size } \\
\text { (US Mesh) }\end{array}$ & -4 & -8 & -14 & -30 & -50 & -100 & -200 & -400 \\
\hline Particle size & -4.75 & -2.36 & -1.40 & -0.600 & -0.300 & -0.150 & -0.075 & -0.038 \\
$(\mathbf{m m})$ & +2.36 & +1.40 & +0.600 & +0.300 & +0.150 & +0.075 & +0.038 & \\
\hline Wt. $\%$ & 21.8 & 10 & 11.5 & 12.6 & 9 & 10.6 & 14.5 & 10 \\
\hline
\end{tabular}

Chemical compositions of the pre-treated charcoal and coke are presented in Table 2.

Table 2. Inorganic concentrations and ash content of pre-treated charcoal and industrial petroleum coke.

\begin{tabular}{ccccccccc}
\hline Material & $\begin{array}{c}\text { Na } \\
(\mathbf{p p m})\end{array}$ & $\begin{array}{c}\mathrm{Ca} \\
(\mathbf{p p m})\end{array}$ & $\begin{array}{c}\mathrm{Si} \\
(\mathbf{p p m})\end{array}$ & $\begin{array}{c}\mathbf{V} \\
(\mathbf{p p m})\end{array}$ & $\begin{array}{c}\mathrm{Fe} \\
(\mathbf{p p m})\end{array}$ & $\begin{array}{c}\text { Ni } \\
(\mathbf{p p m})\end{array}$ & $\begin{array}{c}\mathbf{S} \\
(\mathbf{\%})\end{array}$ & $\begin{array}{c}\text { Ash } \\
(\mathbf{\%})\end{array}$ \\
\hline $\begin{array}{c}\text { Pre-treated charcoal } \\
\text { Pet. Coke }\end{array}$ & 29 & 1685 & 312 & 0 & 58 & 2 & 0 & 0.55 \\
\hline
\end{tabular}

\subsection{Laboratory Pilot Scale Anode Preparation}

Laboratory scale anodes were prepared using petroleum coke aggregates with specific size fractions as listed in Table 1 and coal tar pitch. Only one type of coal tar pitch was used as a binder for all the experiments in this work. Table 3 shows the physical properties and chemical compositions of the pitch. 
Table 3. Physical properties and chemical compositions of the applied pitch.

\begin{tabular}{ccccccccc}
\hline \multirow{2}{*}{$\begin{array}{c}\text { Mettler Softening } \\
\text { Point }\left({ }^{\circ} \mathbf{C}\right)\end{array}$} & $\begin{array}{c}\text { Quinoline } \\
\text { Insoluble (\%) }\end{array}$ & $\begin{array}{c}\text { Coking } \\
\text { Value (\%) }\end{array}$ & \multicolumn{5}{c}{ Chemical Compositions (ppm) } \\
\cline { 5 - 9 } & 16.5 & 58.8 & 93 & Fe & Ca & Na & Si & S (\%) \\
\hline 109.5 & & & & & & & &
\end{tabular}

The reference anodes were made completely of only petroleum coke and pitch. A pitch/coke mass ratio of 16.2/100 was used to produce the samples. For each sample, the pitch and dry coke were placed in the mixing pane and preheated at $178^{\circ} \mathrm{C}$ in an oven (GCA/Precision Scientific, Chicago, IL, USA) for $30 \mathrm{~min}$. The preheated materials were then mixed in a Hobart N50 mixer (Hobart, Troy, $\mathrm{OH}, \mathrm{USA}$ ) for $10 \mathrm{~min}$ to produce the green anode paste. The mixer was installed in the preheating oven to ensure a uniform temperature. The pastes were compacted at $150{ }^{\circ} \mathrm{C}$ in a rigid cylindrical steel mold having an internal diameter of $68 \mathrm{~mm}$. Compaction was performed using $60 \mathrm{MPa}$ uniaxial pressure. The preheating, mixing, pressing, and baking parameters were selected based on the previous works [9].

To produce anodes containing charcoal, $50 \%-100 \%$ of the finest fraction of coke ( -400 mesh) was substituted with the same particle size of the pre-treated charcoal. Two sets of anodes were prepared, having an overall content of $5 \%$ and $10 \%$ of charcoal. Hereafter, the reference anode and anodes containing $5 \%$ and $10 \%$ pre-treated charcoal are named as Ref A, AC-5\%, and AC- $10 \%$ respectively.

\subsection{Optimization of the Anode Recipe}

One of the most important parameters determining the anode paste compaction behavior and the anode density is the pitch/coke ratio. This ratio is usually determined empirically in the anode plants. The rationale for the choice of optimum pitch content is the fact that the pitch should wet the fine coke, providing a binder matrix with sufficient viscosity to enhance the compactability of the anode paste. Thus, the pitch content would depend on the amount of the fine fraction as well as its specific surface area. The Blaine number of the fine coke is usually used as an indication of its specific surface area, and the pitch content is adjusted as a function of the Blaine number.

By substituting the ultra-fine coke with charcoal in the anode recipe, the Blaine number is changed, as should the pitch content. In order to reveal the effect of the pitch content on the anode properties, anode samples with 10\% charcoal and different pitch/coke mass ratios of 16, 18, 20, and 22/100 were prepared. The same coke particle size as presented in Table 1 was used to prepare these samples. The desired amount of pitch was added to the dry aggregate and then mixed and pressed using the same previously described conditions to produce green anodes with different pitch/coke mass ratios. The green apparent density for each sample was measured. All the green anode samples were placed in an Inconel box (Rolled Alloys Inc., Laval, QC, Canada) and covered with coke particles to avoid air burning during the baking process. The anodes were baked in a box furnace using the following baking program: ambient temperature was increased to $150{ }^{\circ} \mathrm{C}$ at a heating rate of $60{ }^{\circ} \mathrm{C} / \mathrm{h}$, then the temperature increased to $650{ }^{\circ} \mathrm{C}$ at $20^{\circ} \mathrm{C} / \mathrm{h}$, then $650{ }^{\circ} \mathrm{C}$ was increased to the maximum baking temperature of $1100^{\circ} \mathrm{C}$ at $50^{\circ} \mathrm{C} / \mathrm{h}$, and this temperature was kept for $20 \mathrm{~h}$. Finally, the furnace was switched off, allowing the samples to cool to room temperature. The baking conditions were selected based on a previous work [12]. After baking, the baked apparent density, the specific electrical conductivity, Young's modulus, and the compressive strength were measured.

\subsection{Characterization Techniques}

\subsubsection{Green and Baked Anode Apparent Density}

When the apparent density of an anode sample is determined, all porosity is included. The sample is weighed and the geometry measured. The apparent density of the specimen is calculated by dividing its mass by its volume. The green and baked anodes were measured according to the ASTM D5502-00 
(2010) [13] standard method. In this method, the height and diameter of the sample is measured at four different points $90^{\circ}$ apart, and the mean value of these measurements is then determined.

\subsubsection{Specific Electrical Resistivity}

This property was measured according to ISO 11713 standard method [14]. In this method, the current is injected through the sample via two steel plates, both placed on springs to apply a pressure of $3 \mathrm{MPa}$. The voltage drop was measured between two points on the sample using two thin pins mounted on a spring to apply the same pressure on each spots. The specific electrical resistivity is defined as

$$
\rho=\frac{U \cdot A}{I \cdot L}
$$

where

$\rho=$ specific electrical resistivity, $\mu \Omega \cdot m$

$U=$ voltage drop over the sample, $\mathrm{V}$

$A=$ cross section of the sample, $\mathrm{m}^{2}$

$L=$ distance between potential contacts, $\mathrm{m}$

$I=$ electrical current through the sample, A

The test was performed eight times for each sample, four times around the sample periphery with a $90^{\circ}$ rotation between each test. For the other four tests, the sample was rotated axially and the measurements were performed in the same way. The average of these eight values was reported as the specific electrical resistivity of the sample.

\subsubsection{Compressive Strength and Young's Modulus}

Compressive strength was measured according to ISO 18515 [15]. A Servo-hydraulic press (MTS Systems, Eden Prairie, MN, USA) was used to perform this test. A linearly increasing compressive force was applied until the sample was crushed. The deformation of the sample (strain) was calculated and the stress-strain curve was plotted. The maximum applied stress on this curve was reported as the compressive strength. Young's modulus was determined from the slope of the stress-strain curve.

\section{Results and Discussion}

Figure 1 shows the apparent density of the green and baked anode samples containing different amounts of charcoal. Both green and baked apparent density decreased as the percentage of charcoal increased. This behavior could be due to the relatively low bulk density of charcoal. The bulk density of charcoal and calcined petroleum coke are 0.41 and $0.95 \mathrm{~g} \cdot \mathrm{cm}^{-3}$, respectively. In addition, as the charcoal has a relatively larger surface area $\left(12 \mathrm{~m}^{2} \cdot \mathrm{g}^{-1}\right)$ than that of calcined coke $\left(5 \mathrm{~m}^{2} \cdot \mathrm{g}^{-1}\right)$, the amount of pitch was probably not optimum to cover all of the fine particles, affecting the viscosity of the binder matrix and its compaction behavior.

Figure 2 shows the effect of charcoal substitution on the specific electrical resistivity and the compressive strength of the anode. Increasing the charcoal in the anode recipe resulted in remarkable deterioration in both electrical and mechanical properties of the baked anodes. This behavior was expected from the baked density results since the baked density directly affects both parameters. 


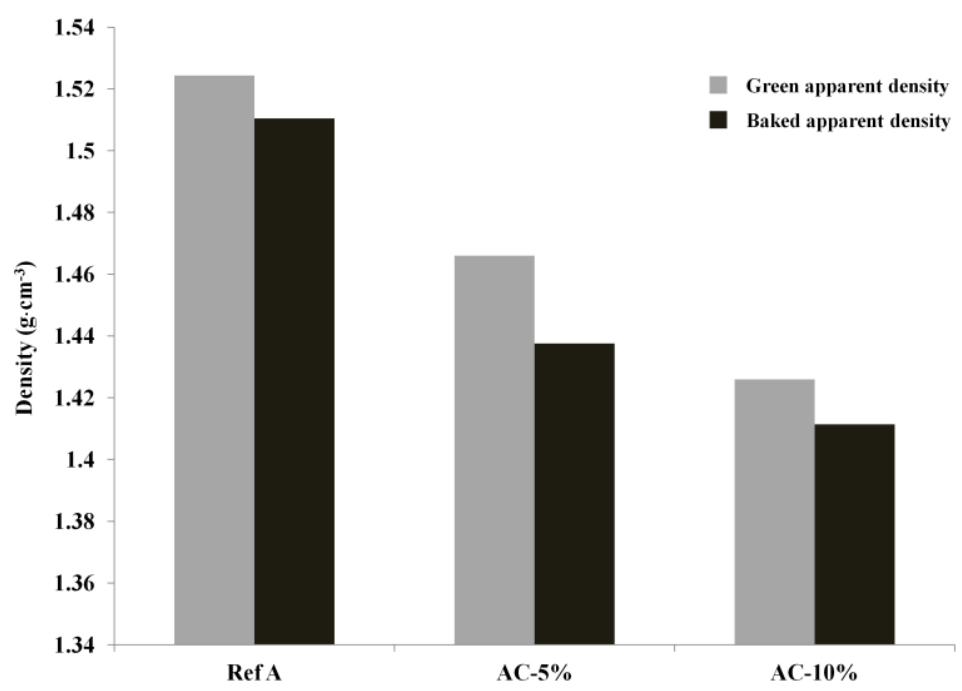

Figure 1. Apparent density for green and baked anodes with different amounts of charcoal.
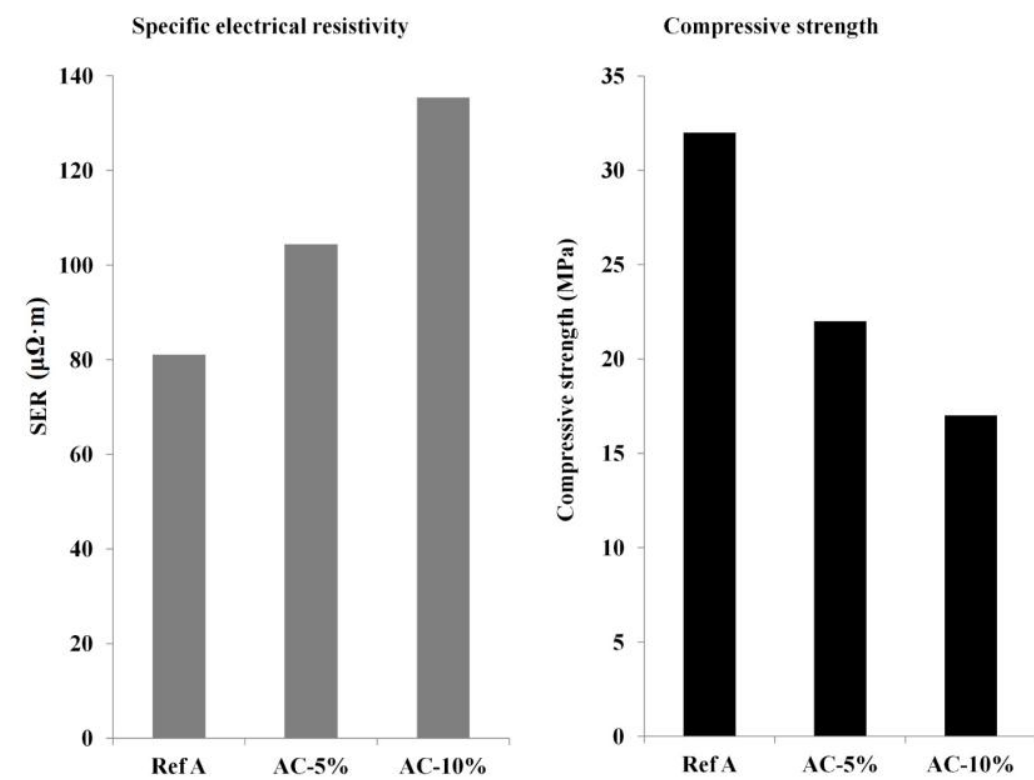

Figure 2. Specific electrical resistivity and compressive strength of anodes with different amounts of charcoal.

\section{Optimization of the Pitch Content}

Figure 3 shows green and baked apparent densities of anodes containing 10\% charcoal but different pitch/coke mass ratios. For comparison, the density of the reference anode is presented in the same figure. By increasing the pitch/coke ratio by 2/100 (i.e., from 16 to 18/100), a pronounced increase in the anode density was achieved. Gradual improvement in the green apparent density was noticed by a further increase in the pitch content, which means that the density approaches a value asymptotically. As expected, this behavior can be attributed to the availability of more pitch for better coverage of the fine particles, providing a suitable binder matrix for compaction. The apparent density of the baked anodes achieves a maximum value by increasing the pitch/coke ratio, and further pitch addition leads to an increased baking loss that reduces the baked density $[9,11]$. Although the negative effect of charcoal on the green and baked density is greatly mitigated by increasing the pitch content, the apparent densities of the anodes containing charcoal are still lower than that of the reference anode. In the best case (22/100 pitch/coke ratio), the density of the baked sample is $3 \%$ lower than that of 
the reference sample. This could be attributed to the low density (high porosity) of charcoal itself compared to that of calcined coke. The pore size of the charcoal is most likely too small to be totally filled with pitch during mixing.

Figure 4 shows the effect of pitch content on the specific electrical resistivity (SER) of the anodes containing $10 \%$ charcoal. As the density of the anode was improved by increasing the pitch content, the specific electrical resistivity was reduced. These results suggest the influence of the anode baked apparent density on its SER. The SER of the anodes experienced reduction by $26.1 \mu \Omega \cdot \mathrm{m}$ as the pitch/coke ratio was increased from 16 to 18/100. Further pitch addition up to 22/100 led to a slight reduction in SER. As the baked anode density approaches a maximum value when pitch content is increased, so does the electrical resistivity. It decreases rapidly at first and then at a much lower rate.

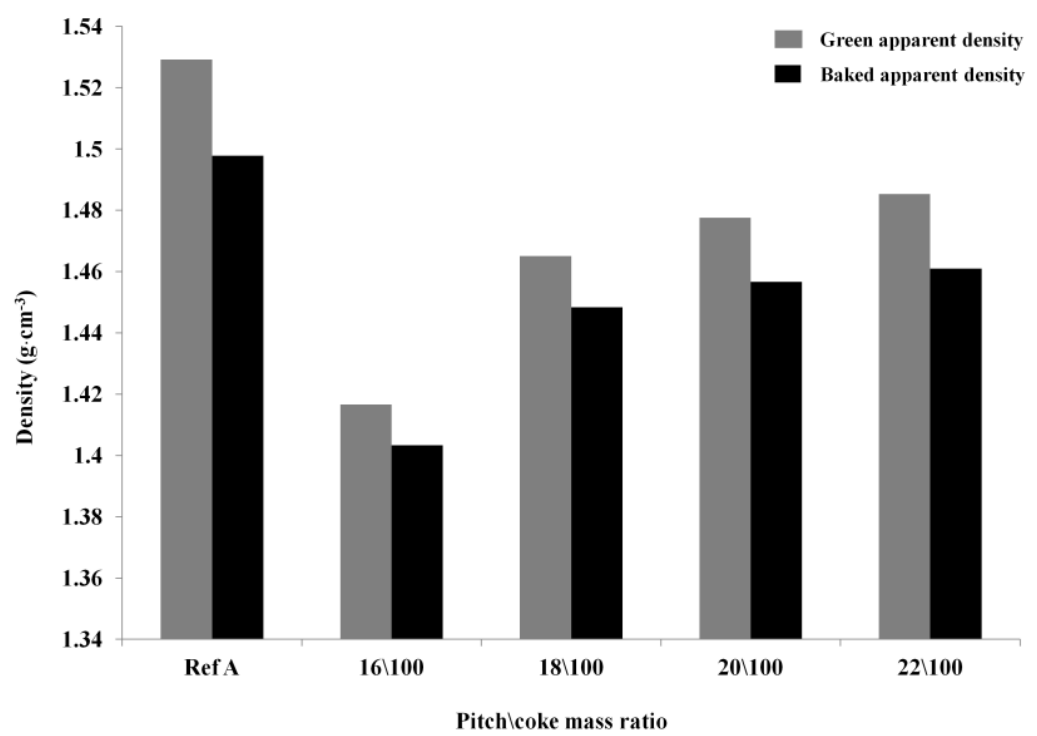

Figure 3. The influence of pitch content on the green and baked apparent density of anodes containing $10 \%$ charcoal compared to the reference anode (Ref A).

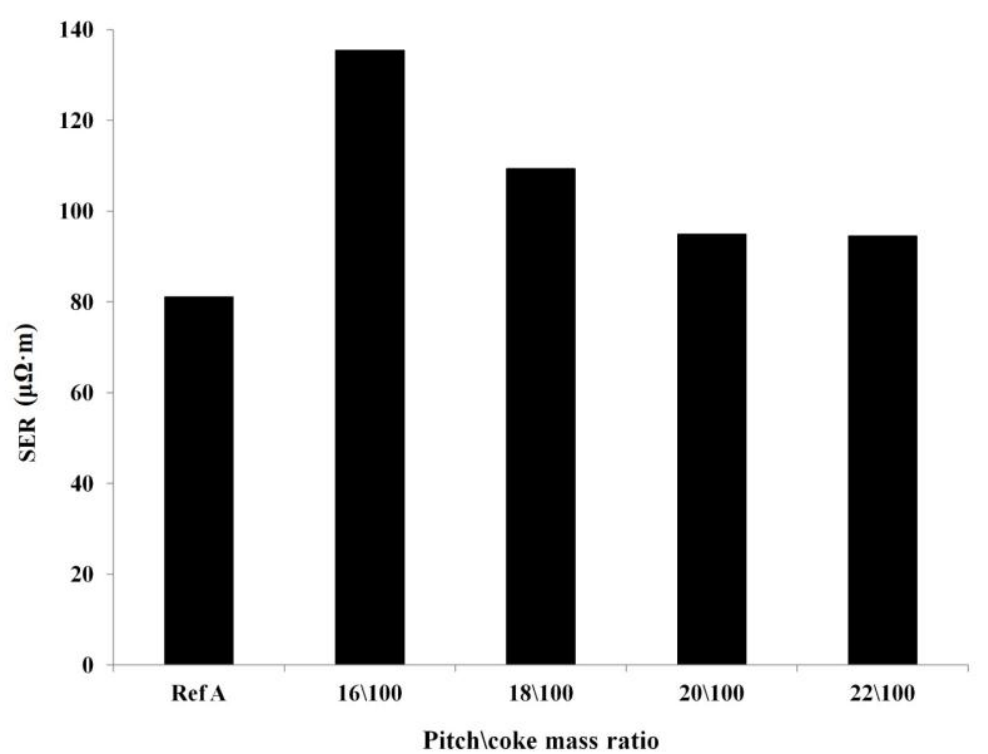

Figure 4. The influence of pitch content on the specific electrical resistivity (SER) of anodes containing $10 \%$ charcoal compared to the reference anode (Ref A). 
Figure 5 shows the effect of pitch content on the mechanical properties of anodes. A significant improvement in both compressive strength and Young's modulus was achieved by increasing the pitch content. It is interesting that the compressive strength and Young's modulus of the baked samples with a 22/100 pitch/coke ratio are similar to those of the reference samples, even though its density is about $3 \%$ lower. These results suggest that the negative effect of charcoal substitution on the mechanical properties of the anode can be fully neutralized by adjusting the pitch content.
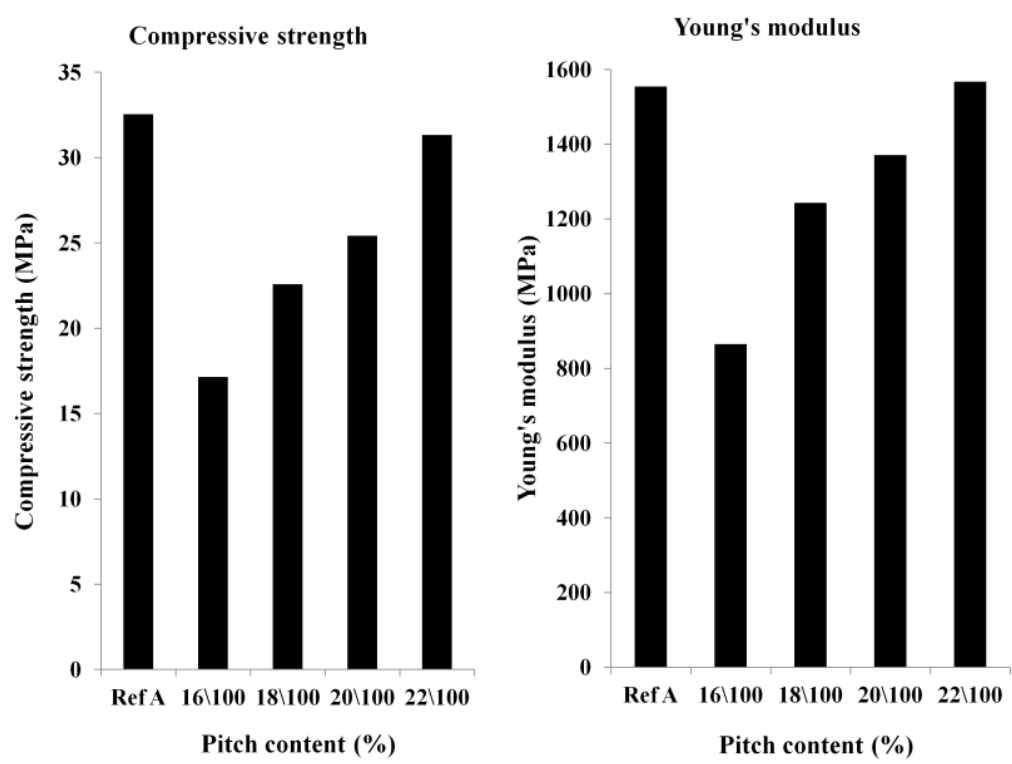

Figure 5. The effect of pitch content on the compressive strength and Young's modulus of anodes containing $10 \%$ charcoal compared to the reference anode (Ref A).

\section{Conclusions}

Using pre-treated charcoal in the anode recipe resulted in a general deterioration in anode properties. Increasing the charcoal content led to a decrease in density, conductivity, and strength of the produced anodes. This behavior was attributed to the relatively large surface area and low bulk density of the charcoal particles. However, increasing the pitch content was found to be helpful for a better covering of charcoal particles with pitch, and the pores might be more filled with pitch as well, resulting in a significant improvement in the physical properties of the anodes containing $10 \%$ charcoal. The best baked anode in this study (22/100 pitch/coke and 10\% charcoal) showed a density of $3 \%$ lower than that of the reference anode, an $18 \%$ higher electrical resistivity, and similar mechanical properties.

Acknowledgments: The authors would like to acknowledge the financial support of National Science and Engineering Research Council of Canada (NSERC) and Alcoa (grant number RDCPJ47654-14). The assistance of Alcoa team (Deschambault plant situated in Deschambault, QC, Canada) is gratefully acknowledged. The authors would also like to extend their appreciation to Guillaume Gauvin and Hugues Ferland for their technical support.

Author Contributions: Asem Hussein and Houshang Alamdari conceived and designed the experiments; Asem Hussein performed the experiments; Mario Fafard, Donald Ziegler and Houshang Alamdari analyzed the data; Asem Hussein wrote the paper and all the co-authors commented and corrected it.

Conflicts of Interest: The authors declare no conflict of interest.

\section{References}

1. Habashi, F. Extractive metallurgy of aluminum. In Handbook of Aluminum; Marcel Dekker: New York, NY, USA, 2003; Volume 2, pp. 1-5.

2. Meier, M.W. Cracking Behaviour of Anodes. Ph.D. Thesis, Federal Institute of Technology, Zurich, Switzerland, 3 January 1996; pp. 12-40. 
3. Edwards, L. The History and Future Challenges of Calcined Petroleum Coke Production and Use in Aluminum Smelting. JOM 2015, 67, 308-321. [CrossRef]

4. Jahirul, M.; Rasul, M.; Chowdhury, A.; Ashwath, N. Biofuels Production through Biomass Pyrolysis-A Technological Review. Energies 2012, 5, 4952-5001. [CrossRef]

5. Monsen, B.; Ratvik, A.P.; Lossius, L.P. Charcoal in anodes for aluminium production. TMS Light Met. 2010, 2010, 929-933.

6. Elkasabi, Y.; Boateng, A.A.; Jackson, M.A. Upgrading of bio-oil distillation bottoms into biorenewable calcined coke. Biomass Bioenergy 2015, 81, 415-423. [CrossRef]

7. Coutinho, A.R.; Rocha, J.D.; Luengo, C.A. Preparing and characterizing biocarbon electrodes. Fuel Process. Technol. 2000, 67, 93-102. [CrossRef]

8. Hussein, A.; Larachi, F.; Zeigler, D.; Alamdari, H. Effects of heat treatment and acid washing on properties and reactivity of charcoal. Biomass Bioenergy 2016, 90, 101-113. [CrossRef]

9. Azari, K.; Alamdari, H.; Aryanpour, G.; Ziegler, D.; Picard, D.; Fafard, M. Compaction properties of carbon materials used for prebaked anodes in aluminum production plants. Powder Technol. 2013, 246, 650-657. [CrossRef]

10. Smith, M.A. An Evaluation of the Binder Matrix in Prebaked Carbon Anodes Used for Aluminium Production. Ph.D. Thesis, University of Auckland, Auckland, New Zealand, April 1991.

11. Hulse, K.L. Anode Manufacture: Raw Materials, Formulation and Processing Parameters, 1st ed.; R \& D Carbon Ltd.: Sierre, Switzerland, 2000; pp. 77-158.

12. Chevarin, F.; Lemieux, L.; Picard, D.; Ziegler, D.; Fafard, M.; Alamdari, H. Characterization of carbon anode constituents under $\mathrm{CO}_{2}$ gasification: A try to understand the dusting phenomenon. Fuel 2015, 156, 198-210. [CrossRef]

13. ASTM International. ASTM D5502-00(2010), Standard Test Method for Apparent Density by Physical Measurements of Manufactured Anode and Cathode Carbon Used by the Aluminum Industry; ASTM International: West Conshohocken, PA, USA, 2010.

14. International Organization for Standardization. ISO 11713, Standard Method, Carbonaceous Materials Used in the Production of Aluminium-Cathode Blocks and Baked Anodes-Determination of Electrical Resistivity at Ambient Temperature; International Organization for Standardization: Geneva, Switzerland, 2000.

15. International Organization for Standardization. O 18515, Carbonaceous Materials for the Production of Aluminium-Cathode Blocks and Baked Anodes-Determination of Compressive Strength; International Organization for Standardization: Geneva, Switzerland, 2014.

(C) 2017 by the authors. Licensee MDPI, Basel, Switzerland. This article is an open access article distributed under the terms and conditions of the Creative Commons Attribution (CC BY) license (http:/ / creativecommons.org/licenses/by/4.0/). 